Public Health

\section{Visceral leishmaniasis}

\section{in Afghanistan}

$\mathrm{D}$ espite being the third most important vector-borne diseases worldwide in terms of burden of disease, the leishmaniases are one of the so-called "neglected diseases."

Since the massive humanitarian reconstruction efforts began after the fall of the Taliban in 2002, Afghanistan is slowly emerging from 2 decades of civil war. Nevertheless, country health indicators are still among the worst globally, with many preventable diseases such as typhoid fever, diarrhea and measles common throughout the country. ${ }^{1}$ Besides having the world's largest incidence of cutaneous leishmaniasis, with an estimated 250000 cases o annually, ${ }^{2}$ Afghanistan may now have another public health problem on their hands: visceral leishmaniasis.

Epidemiology: Visceral leishmaniasis is a chronic, systemic disease characterized by fever, (hepato)splenomegaly, lymphadenopathy, pancytopenia, weight loss, weakness and, if left untreated, death. ${ }^{3}$ The disease is usually caused by Leishmania donovani or L. infantum, transmitted to human and animal hosts by the bite of phlebotomine sandflies. Although in most diseaseendemic areas transmission is zoonotic, with domestic dogs as the main reservoir, transmission is anthroponotic in some areas, such as India and Sudan.

Clinical management: In most areas where visceral leishmaniasis is endemic, suspected cases are diagnosed on the basis of positive Giemsa-stained smears of lymph node, bone marrow or spleen aspirates. ${ }^{3}$ Recently, there also has been an increased operational use of easy-to-use serologic tests (e.g., immunochromatographic dipsticks or direct agglutination tests). Following parasitologic confirmation, patients are usually given pentavalent antimony therapy $(20 \mathrm{mg} / \mathrm{kg}$ intramuscularly or intravenously) for 30 days. ${ }^{3}$ Other therapeutic approaches include miltefosine and amphotericin $B$, especially in disease-endemic areas where increased unresponsiveness to antimony has been observed.
Prevention: Strategies to prevent and control visceral leishmaniasis are aimed at reducing exposure to the sandfly vector. This can be achieved by improving housing, using insecticidetreated bednets or curtains, spraying houses indoors with residual insecticides and - in zoonotic foci - collaring dogs with insecticide-impregnated dog collars. Although these strategies have proven to be highly effective, they have usually been implemented only on a foci-specific scale rather than nationally or regionally.

Visceral leishmaniasis in Afghanistan: Although several hundreds of cases of visceral leishmaniasis are reported every year from Afghanistan's neighbouring countries (Iran, Pakistan, Turkmenistan and Uzbekistan), there have been only 23 cases reported from Afghanistan since 1980 in the scientific literature.

Recently, 6 parasitologically confirmed cases of visceral leishmaniasis in patients from 3 Afghan provinces were observed in HealthNet International's leishmaniasis treatment centre in Kabul. The patients' demographic and clinical characteristics are given in Table $\mathrm{I}$. This increased detection in the

Table 1: Demographic and clinical characteristics of 6 cases of visceral leishmaniasis in Afghan patients referred to HealthNet International's clinic in Kabul between February and April 2005

\begin{tabular}{|c|c|c|c|c|c|}
\hline Case & Age, yr & Sex & Residence & Clinical history and presentation & Date of infection* \\
\hline 1 & 8 & M & Gorzai Village, Baghlan & $\begin{array}{l}\text { Malnutrition and incomplete child immunization; } \\
\text { hepatosplenomegaly and intermittent fever for } \\
18 \text { mo; no history of travel in year before } \\
\text { presentation; initial diagnosis of malaria }\end{array}$ & Autumn 2003 \\
\hline 2 & 10 & M & Borka Village, Baghlan & $\begin{array}{l}\text { Gross splenomegaly, intermittent fever for } 18 \mathrm{mo} \text {; } \\
\text { gradual weight loss; several episodes of confirmed } \\
\text { Plasmodium vivax malaria }\end{array}$ & Autumn 2003 \\
\hline 4 & 7 & $\mathrm{~F}$ & Arabkhana Village, Faryab & $\begin{array}{l}\text { Hepatosplenomegaly, intermittent fever for } 7 \mathrm{mo} \text {; } \\
\text { no history of travel in year before presentation; } \\
\text { initial diagnosis of malaria and typhoid }\end{array}$ & Summer 2004 \\
\hline
\end{tabular}

Note: In 5 cases the original diagnosis was malaria, and the patients were presumptively given treatment with chloroquine ( $25 \mathrm{mg} / \mathrm{kg}$ ). Giemsa-stained slides of bone marrow aspirates from all patients were microscopically positive for Leishmania parasites; all 6 cases were retrospectively confirmed by means of polymerase chain reaction to have been caused by $L$. donovani. All cases were successfully treated with intramuscularly administered sodium stibogluconate ( $20 \mathrm{mg} / \mathrm{kg}$ daily) for 21 days according to Afghan treatment guidelines.

*Estimated from patient's history and season of leishmaniasis transmission. 


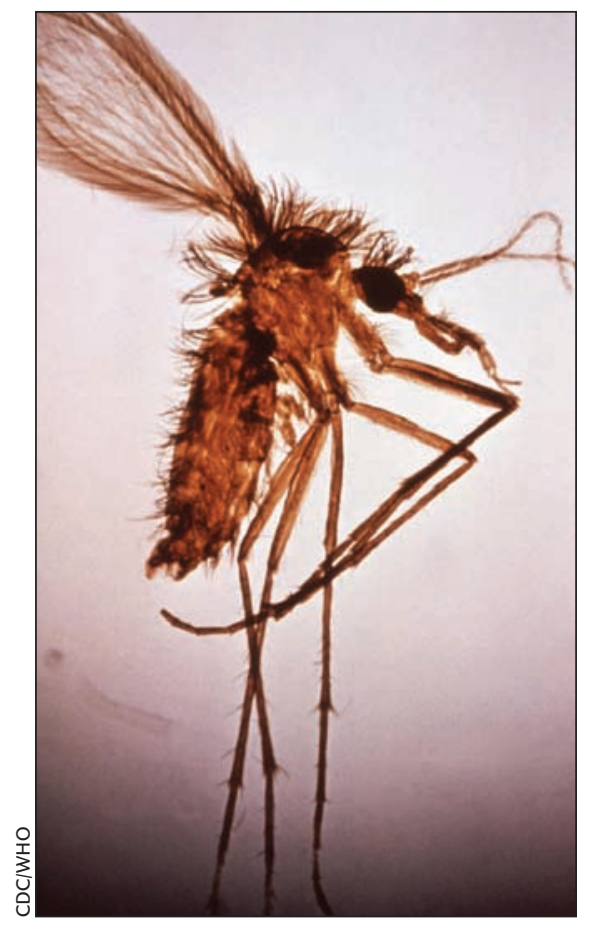

Female phlebotomine sandfly. Sometimes mistaken for mosquitoes, these sandflies are common vectors for various diseases, including leishmaniasis.

number of cases is of concern, since it follows the report of 2 US soldiers stationed in Afghanistan with the disease ${ }^{4}$ and 2 cases from Baghlan province in 2004 (unpublished data from HealthNet International, Kabul).

Because climate restricts the sandfly vector's season in Afghanistan from April to October, it is probable that the patients, given their individual case histories, contracted the disease in 2004 or 2003 . Most of the cases reported here were from the northeastern province of Baghlan. They could have resulted from a recent outbreak or, most probably, be coincident cumulative reporting of a disease with low endemicity (e.g., owing to better access to health care). Of interest is that most of the patients had originally received a diagnosis of malaria, which is endemic throughout Afghanistan.

There is little historical or current data on visceral leishmaniasis in Afghanistan: neither vectors nor reservoirs have been incriminated, and it is unknown whether the transmission cycle is anthroponotic or zoonotic. Epidemiologic research is highly warranted. The increased detection of visceral leishmaniasis in Afghanistan will require a reassessment of leishmaniasis prevention and control strategies. Unlike cutaneous leishmaniasis, the visceral form is potentially fatal, with case-fatality rates as high as $85 \%$. Large-scale occurrence of a potentially fatal disease in a population that is largely malnourished and impoverished would be devastating. Leishmaniasis management guidelines in Afghanistan will have to be updated to include available treatment regimens for the visceral form of the disease. Additional prevention and control measures, such as the reduction of animal reservoirs (if any), the introduction and upscaling of personal protection and vector control, awareness campaigns, and training for health professionals, will require significant input from international funding agencies, above and beyond what is currently available for cutaneous leishmaniasis control.

\section{Toby Leslie \\ Sarah Saleheen \\ Mohammad Sami \\ Ismail Mayan \\ Najibullah Mahboob \\ Kathy Fiekert \\ Annick Lenglet \\ Rosalynn Ord \\ Richard Reithinger}

From the Department of Infectious and Tropical Diseases, London School of Hygiene and Tropical Medicine, London, UK (Leslie, Ord); HealthNet International, Kabul, Afghanistan (Saleheen, Sami, Mayan, Fiekert, Lenglet, Reithinger); and the Ministry of Public Health, Kabul, Afghanistan (Mehboob, Fiekert)

Acknowledgements: HealthNet International's Malaria and Leishmaniasis Control Programme is funded by the European Commission (DGI). The views expressed herein are solely those of the authors and do not necessarily reflect those of their respective organizations, institutions or funding agencies. The principal investigator, Toby Leslie, had full access to all of the data in the study and takes responsibility for the integrity of the data and the accuracy of the data analysis.

\section{REFERENCES}

I. Waldmann R, Hanif $\mathrm{H}$. The public health system in Afghanistan: current issues. Islamabad: Afghanistan Research and Evaluation Unit; 2002. Available: www.areu.org.af/publications/waldman _health.pdf (accessed 2006 Jun or).

2. Reithinger R, Mohsen M, Aadil K, et al. Anthroponotic cutaneous leishmaniasis, Kabul, Afghanistan. Emerg Infect Dis 2003;9:727-9.

3. Division of Control of Tropical Diseases, World Health Organization (WHO). Manual on visceral leishmaniasis control. Geneva: WHO; I996. Available: www.who.int/leishmaniasis/surveillance /training/en/WHO_LEISH_96.40.pdf (accessed 2006 Jun I).

4. Two cases of visceral leishmaniasis in U.S. military personnel - Afghanistan, 2002-2004. MMWR Morb Mortal Wkly Rep 2004;53:265-8.

\section{IMPACT}

CMAJ is a leading international general medical journal as ranked by Thomson ISI. 2005 impact factor, 7.4. 